\title{
LASER GUIDE STAR ADAPTIVE OPTICS: PRESENT AND FUTURE
}

\author{
S. S. OLIVIER and C. E. MAX \\ Institute of Geophysics and Planetary Physics \\ Lawrence Livermore National Laboratory \\ P.O. Box 808, Livermore, CA 94551, USA
}

\begin{abstract}
Feasibility demonstrations using one to two meter telescopes have confirmed the utility of laser beacons as wavefront references for adaptive optics systems. Laser beacon architectures suitable for the new generation of eight and ten meter telescopes are presently under study. This paper reviews the concept of laser guide star adaptive optics and the progress that has been made by groups around the world in implementing such systems. A description of the laser guide star program at LLNL and some experimental results is also presented.
\end{abstract}

\section{Introduction}

The concept of using a laser guide star to provide a wavefront reference for adaptive optics systems was proposed independently in the classified literature by Happer in 1982 (Happer et al. 1993) and in the open literature by Foy and Labeyrie (1985). The idea is to use a laser projected upward from the ground to illuminate some region of the atmosphere, and then to use the light from that laser-created spot as a wavefront reference.

Two schemes for creating laser guide stars have been suggested: (1) to use the Rayleigh scattering of UV or visible light from $\sim 5$ to $15 \mathrm{~km}$ altitude, (2) to use resonant scattering of $589 \mathrm{~nm}$ light from the mesospheric sodium layer at $\sim 100 \mathrm{~km}$ altitude. This second scheme has the advantage that the higher reference beacon samples a larger portion of the optical path traversed by light from an astronomical source.

Once a laser guide star has been created, it can be used as a wavefront reference for an adaptive optics system which corrects the aberrations introduced by the atmosphere into images of astronomical objects. A schematic diagram of such a laser guide star adaptive optics system is shown in Figure 1. Light from both the laser guide star and the astronomical object enter the telescope 
and pass through a collimating lens. The wavefront is then reflected from a tip-tilt mirror which compensates for the image motion and a deformable mirror which compensates for higher order wavefront aberrations. The use of a separate tip-tilt mirror reduces the dynamic range requirements for the deformable mirror. The light from the laser guide star and the astronomical object is then separated by a dichroic splitter. The laser guide star light is sent to a wavefront sensor which in turn drives the deformable mirror, while the light from the astronomical object is sent both to a tip-tilt sensor which is used to drive the tip-tilt mirror, and to the imaging camera which records the compensated astronomical image.

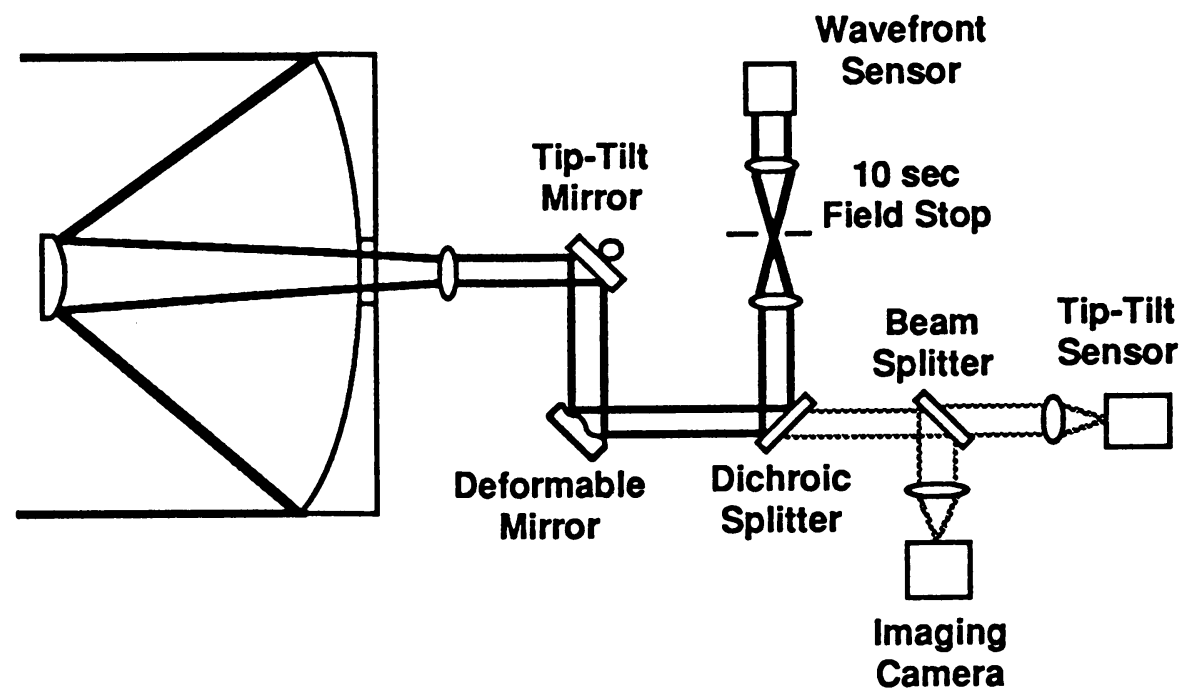

FIGURE 1. Laser guide star adaptive optics system schematic diagram.

Of course, it is only necessary to use a laser guide star when there is no bright natural star close enough to the astronomical object to use as a wavefront reference. In general, one needs a wavefront reference within the isoplanatic angle of the astronomical object, that is the angle over which the atmospheric turbulence is well correlated. Since the isoplanatic angle (and the atmospheric coherence length) scales with wavelength, it has generally been found theoretically that for imaging in the IR, good compensation can be achieved for a large fraction of astronomical objects using natural stars, while for imaging in the visible, laser guide stars are required 
to get reasonable sky coverage. The precise wavelength at which it becomes necessary to use laser guide stars is not well defined; however, recent work (e.g., Olivier 1993) indicates that laser guide stars are needed for wavelengths shorter than $\sim 2 \mu \mathrm{m}$.

Even with a laser guide star, the sky coverage is not complete since the laser guide star cannot be used as a tip-tilt reference. This is due to the fact that the laser is propagated up through the atmosphere before forming the guide star, and thus the absolute position of the laser guide star is not known. Therefore, a natural star must be used as a tip-tilt reference, and there is once again an issue of sky coverage. Fortunately, the isoplanatic angle for tip-tilt is quite a bit larger than for the higher order aberrations. In addition, tip-tilt from a natural star can be sensed using the entire aperture of the telescope. Therefore, even for imaging in the visible, there is usually a reasonably good tip-tilt reference star available. However, the quality of the tip-tilt correction does place an ultimate limit on the long-exposure resolution achievable with laser guide star systems (Olivier et al. 1993, Olivier and Gavel 1993).

Another limitation of laser guide star systems is due to the finite height of the laser guide star. Errors are due to both turbulence from layers above the height of the laser guide star and turbulence in layers below the laser guide star and inside the cylinder extending through the atmosphere from the telescope entrance pupil, but outside the cone extending from the guide star to the telescope entrance pupil. Both of these effects can be mitigated by using the highest possible guide star. In addition, the second effect can be be mitigated by using multiple guide stars.

Taking these limitations on laser guide stars into account, rough practical limits on the utility of Rayleigh and sodium laser guide stars can be determined. Rayleigh guide stars are typically generated at heights of 5 to $20 \mathrm{~km}$ by lasers with wavelengths from green (e.g., copper vapor lasers) to UV (e.g., excimer lasers). For imaging in the visible, one Rayleigh guide star can be used for telescope apertures up to $\sim 2 \mathrm{~m}$. Telescopes with 8 to $10 \mathrm{~m}$ apertures would need tens of Rayleigh laser guide stars, and even with this many spots unsensed turbulence above the Rayleigh scattering altitude limits the quality of correction.

Sodium guide stars are generated at a height of 95 to $105 \mathrm{k}$ - 
$\mathrm{m}$ in the mesospheric sodium layer by lasers tuned to the sodium $D_{2}$ transition at a wavelength of $589 \mathrm{~nm}$. The sodium laser guide star is high enough that uncorrected turbulence above it is not a problem, and one guide star is adequate to correct an 8 to $10 \mathrm{~m}$ telescope for imaging at wavelengths above $\sim 2 \mu \mathrm{m}$. For imaging at visible wavelengths, $\sim 10$ sodium guide stars would be needed for 8 to $10 \mathrm{~m}$ telescopes.

The remainder of this paper will be organized as follows. In $\S$ 2, we will present a review of previous and current laser guide star projects. A more detailed description of the LLNL laser guide star project will be given in $\S 3$. A summary and conclusions will be given in $\S 4$.

\section{Review of Laser Guide Star Projects}

Laser guide stars are being developed by several groups around the world.

The MIT Lincoln Laboratory in Cambridge, Massachusetts performed the first sodium guide star wavefront measurements using two subapertures, and a solid-state laser based on summing the frequency of light from two flash lamp pumped Nd-YAG lasers, tuned to different wavelengths, in a non-linear crystal (Humphries et al. 1991). They also demonstrated optical pumping of the sodium line using circularly polarized light to increase the emission from the sodium atoms.

Using a Rayleigh guide star, the group at Lincoln Lab demonstrated wavefront correction on a single-shot or "go-to" basis with a 241 channel adaptive optics system (Primmerman et al. 1991). They also performed the first experiment with more than one laser beacon.

The Air Force Phillips Laboratory in Albuquerque, New Mexico has demonstrated closed-loop correction with a 241 channel adaptive optics system using a Rayleigh guide star (Fugate et al. 1991). In addition, they have generated a sodium laser guide star using a summed-frequency Nd-Yag laser built by Lincoln Lab.

Thermo-Electron Technologies in San Diego, California has generated single and multiple Rayleigh guide stars using excimer lasers (Sandler 1992). They have also developed a system using a 500 subaperture segmented mirror and a Rayleigh guide star generated with a frequency doubled Nd-YAG laser (Sandler et al. 1992). 
A group at the University of Illinois (Champaign-Urbana) has generated a Rayleigh guide star using an excimer laser and measured the guide star return flux as a function of backscatter altitude (Thompson and Castle 1992). They also performed the first unclassified tests of a sodium laser guide star (Thompson and Gardner 1987).

A group at the University of Chicago (Illinois) has generated a sodium laser guide star using a CW dye laser (Kibblewhite et al. 1992).

A group in France has generated a Rayleigh guide star using a Nd-YAG laser (Foy et al. 1989).

\section{LLNL Laser Guide Star Project}

The LLNL laser guide star project uses a laser developed for atomic vapor laser isotope separation (AVLIS) at LLNL. This laser consists of 12 copper vapor lasers that make green light that is then used to pump dye lasers which can be tuned to the sodium $D_{2}$ line. The average power from this laser is $\sim 1.5 \mathrm{~kW}$ at $589 \mathrm{~nm}$ and at a pulse repetition rate of $26 \mathrm{kHz}$, making it the most powerful laser by a factor of $\sim 100$ ever used to generate a sodium laser guide star. The laser has the capability to modulate its line shape so as to optimize the return flux from the sodium layer. In addition, the high beam quality of the laser ( 1.5 diffraction limited) allows propagation with low divergence losses. Finally, since the laser was engineered for factory use, it is extremely reliable making it a credible "utility" for an observatory.

Using the AVLIS laser, a sodium laser guide star was generated at LLNL and the return flux was measured as a function of laser power. The experimental results are shown in Figure 2. Also shown in Figure 2 is a theoretical prediction that was calculated using a 24 level Bloch model for the atomic physics of the sodium atoms (Morris 1993). The agreement between the model prediction and the data is quite good (Avicola, et al. 1993).

In order to demonstrate closed-correction with the sodium laser guide star, an adaptive optics system is now under development at LLNL. This system will be mounted on a $0.5 \mathrm{~m}$ telescope located $5 \mathrm{~m}$ from the laser beam director.

The wavefront from the sodium laser guide star will be measured with a Hartmann sensor based on a Kodak intensified CCD 


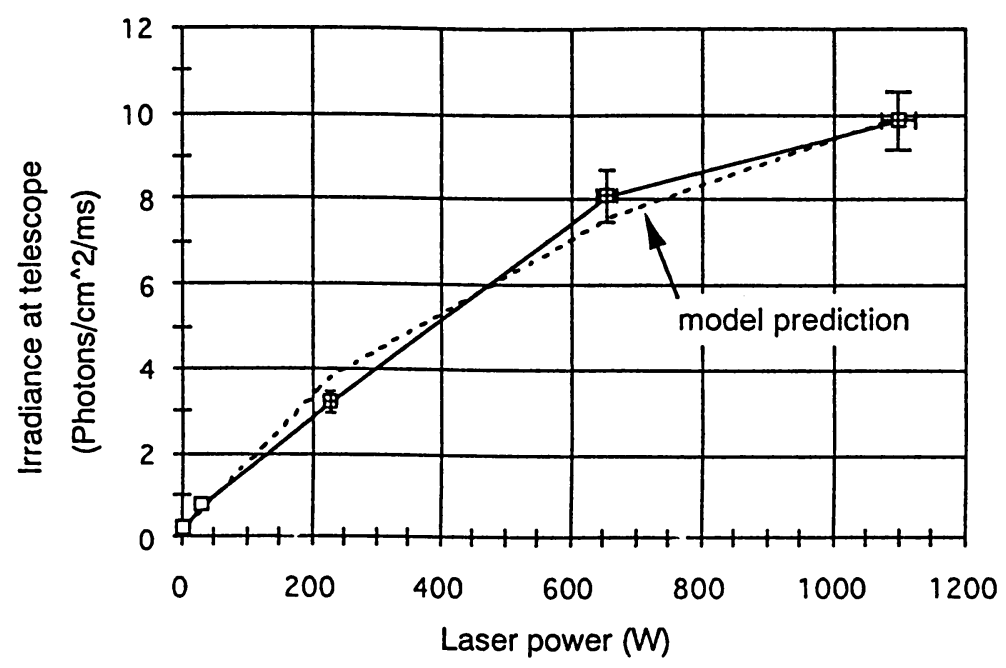

FIGURE 2. Return flux from LLNL sodium laser guide star as a function of laser power.

camera. This camera has a large format $(192 \times 239$ pixels $)$, a high frame rate $(1 \mathrm{kHz})$, but a low quantum efficiency $(5 \%$ at $589 \mathrm{n}$ $\mathrm{m})$. In the future, the Kodak camera will likely be replaced with a high quantum efficiency, low noise, fast CCD camera. The Kodak wavefront sensor has been tested on the telescope both with natural stars and with the sodium laser guide star. Figure 3 shows an average of 100 frames of Kodak wavefront sensor data from both a natural star and the sodium laser guide star.

The wavefront reconstructor will be based on four Intel i860 chips on a VME board and will be controlled by a Unix workstation. The wavefront reconstructor has been tested using data from the wavefront sensor obtained in tests on the telescope.

The deformable mirror design is based on mirrors used for beam control in the AVLIS program. It will have a triangular array of 19 actuators and a continuous face sheet. The mirror has been fabricated and is currently being tested in the laboratory.

The tip-tilt system will be based on an avalanche photodiode quad-cell sensor and a small $(2.5 \mathrm{~cm})$ tip-tilt mirror with an analog controller. The closed-loop operation of this system has been tested in the laboratory.

Because the AVLIS laser has short pulses and high average 

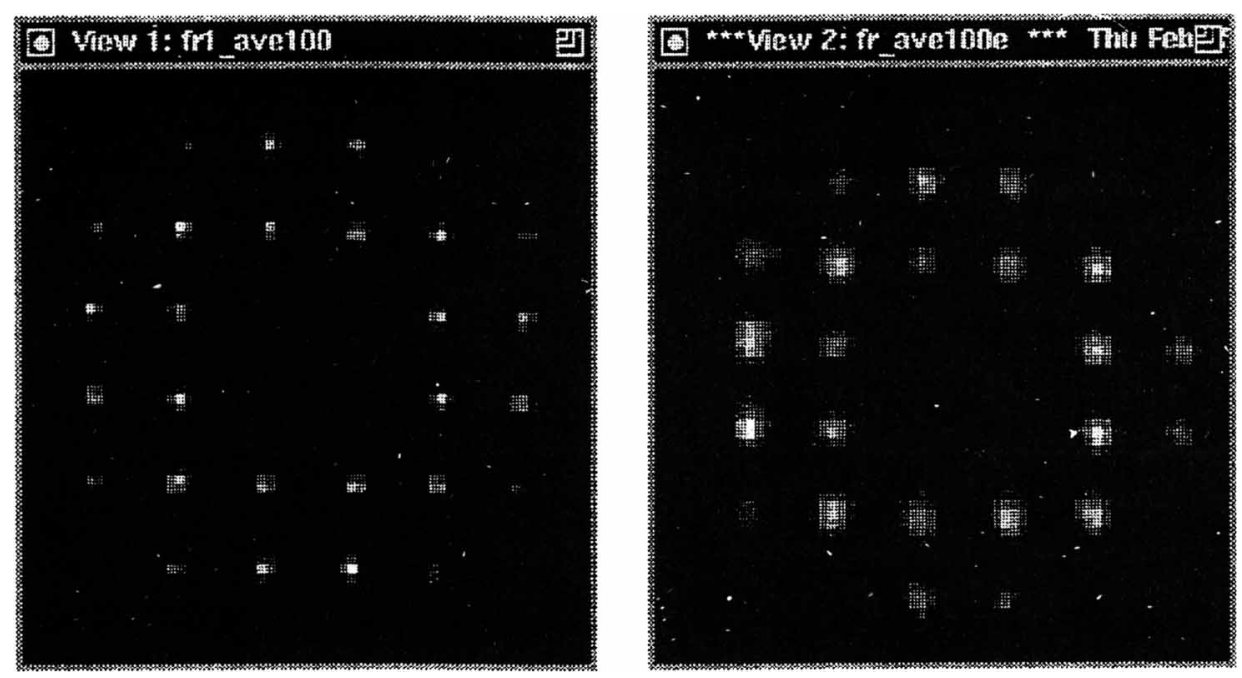

S-01230184 DAR_VG022593_1

FIGURE 3. Kodak wavefront sensor data. The left panel shows the average of 100 frames from a natural star. The right panel shows the average of 100 frames from the LLNL sodium laser guide star.

power, the peak power of each pulse saturates the sodium layer.

This is the cause of the rolloff in return flux at high power seen in Figure 2. In order to get better performance at high power an optical pulse stretcher is being developed to lengthen each pulse by a factor of 16. The pulse stretcher has been tested at low power and functions well.

Closed-loop demonstration of the LLNL laser guide star adaptive optics system is scheduled for the Fall of 1993. In addition, a 69 subaperture adaptive optics system is being built by LLNL as a prototype system for the Lick Observatory, and preliminary design studies for adaptive optics at the Keck Observatory and for laser guide star systems at both Lick and Keck Observatories are being performed.

\section{Summary and Conclusions}

Laser guide star adaptive optics are a reality. For example, Rayleigh guide stars have been used for closed-loop atmospheric turbulence compensation with 241 subapertures on a $1.5 \mathrm{~m}$ telescope (Phillip$\mathrm{s}$ Laboratory). Sodium guide stars are somewhat behind, but are 
catching up. For example, the LLNL AVLIS laser has been used to create a $V=5$ laser guide star. This will be more than adequate for closed-loop adaptive optics compensation.

The crucial next step will be to demonstrate the utility of laser guide stars at an astronomical observatory. It must be shown that laser guide star adaptive optics can be used to produce exciting scientific results. In addition, it is important to demonstrate that laser guide stars are not too much trouble to use at an observatory.

Finally, theoretical predictions of laser guide star adaptive optics performance on the largest telescopes look very favorable, in part because these telescopes tend to be located at excellent seeing sites. Indeed, the future of laser guide stars would appear to be very bright!

Work performed under the auspices of the U.S. Department of Energy by Lawrence Livermore National Laboratory under Contract W-7405-Eng-48.

\section{References}

Avicola, K., et al. 1993, J. Opt. Soc. Am. A, in press

Foy, R., \& Labeyrie A. 1985, A\&A, 152, L29

Foy, R., Tallon, M., Séchaud, M., \& Hubin, N. 1989, in Active Telescope Systems, ed. F. J. Roddier, (Proc. SPIE, vol. 1114), 174

Fugate, R. Q., et al. 1991, Nature, 353, 144

Happer, W., MacDonald, G., Max, C., \& Dyson, F. 1993, J. Opt. Soc. Am. A, in press

Humphreys, R. A., Primmerman, C. A., Bradley, L. C., \& Herrmann, J. 1991, Opt. Lett., 16, 1367

Kibblewhite, E., et al. 1992, in Proc. Laser Guide Star Adaptive Optics Workshop, ed. R. Q. Fugate (Albuquerque: Phillips Lab), 522

Morris, J. R. 1993, J. Opt. Soc. Am. A, in press

Olivier, S. S. 1993, ApJ, in preparation

Olivier, S. S. \& Gavel, D. T. 1993, J. Opt. Soc. Am. A, in press

Olivier, S. S., Max, C. E., Gavel, D. T., \& Brase, J. M. 1993, ApJ, in press

Primmerman C. A., Murphy, D. V., Page, D. A., Zollars, B. G., \& Barclay, H. T. 1991, Nature, 353, 141 
Sandler, D. 1992, in Proc. Laser Guide Star Adaptive Optics Workshop, ed. R. Q. Fugate (Albuquerque: Phillips Lab), 164 Sandler, D., et al. 1992, in Proc. Laser Guide Star Adaptive Optics Workshop, ed. R. Q. Fugate (Albuquerque: Phillips Lab), 686

Thompson, L. A., \& Castle, R. M. 1992, Opt. Lett., 21, 1485

Thompson, L. A., \& Gardner, C. S. 1987, Nature, 328, 229

\section{Discussion:}

\section{Roddier:}

What is the angular size of your laser guide star?

Olivier:

At full power (i.e. $\sim 1.5 \mathrm{kw}$ ), the guide star has an angular size of $\sim 8$ arsec.

\section{Baldwin:}

What determines the 8 arcsec size of the laser guide star in your experiments?

Olivier:

The beam is projected from a $4 \times 8 \mathrm{~cm}$ aperture which is oriented so that the elongation due to the $5 \mathrm{~m}$ separation between the beam director and the telescope compensates for the rectangular beam format, and the observed image is nearly round. The $4 \mathrm{~cm}$ projecting aperture size along with the atmosphere would normally limit the angular size to $\sim 4 \operatorname{arcsec}$. The factor of 2 which gives the 8 arsec size is due to the fact that at high power our laser saturates the sodium layer, thereby bringing up the wings of the spot relative to the center.

\section{Radhakrishnan:}

An angular size of $8^{\prime \prime}$ gives a linear spot size of $\sim 4 \mathrm{~m}$ at the sodium layer, i.e. not much smaller than the telescope. So I find your cone diagram misleading.

Olivier:

Our expectation is that under normal operation in an astronomical setting, one would not use a laser which saturated the sodium layer. Then, under good seeing conditions, one might obtain a spot size of $\sim 2^{\prime \prime}$ or $\sim 1 \mathrm{~m}$ at the sodium layer. This size would be significantly smaller than a large telescope, and the cone diagram would then be valid. 
Eckart:

How well does the correction apply in the optical even though the size of the artifical star $\left(8^{\prime \prime}\right)$ is larger than the isoplanatic patch $\left(3^{\prime \prime}-5^{\prime \prime}\right)$ ?

Olivier:

Saśiela (Lincoln Lab Tech. Rep. No. 807, 1988) has calculated the anisoplanatic estimation error due to finite spot size as $\sigma^{2}=\left(0.1 \theta_{*} / \theta_{0}\right)^{\frac{5}{3}}$, where $\theta_{*}$ is the size of the guide star and $\theta_{0}$ is the isoplanatic angle. Using this formula, one obtains a Strehl ratio of $\sim 0.93$ if $\theta_{*}=8$ arcsec and $\theta_{0}=4 \mathrm{arcsec}$. In the visible, $\theta_{0}$ is probably closer to 2 arcsec which would give a Strehl ratio of $\sim 0.80$ for an 8 arcsec guide star.

Ekers:

If your laser spot is $8^{\prime \prime}$ in size how do you measure the Fourier component corresponding to wavefront errors over large separations in the aperture?

Olivier:

The laser spot is used to measure the average phase gradient of the incoming wavefront in many small subapertures over the full telescope aperture. This is done by measuring the position of the image of the spot as seen by each subaperture. The wavefront is then reconstructed by interpolating the phase measurements from the subaperture grid. Since there is no attempt to form an interferogram of the spot between distant subapertures, there is no problem with the $8 \operatorname{arcsec}$ spot size. The only penalty in this context is a loss of sensitivity as the spot becomes resolved in a subaperture.

Masson:

How much power does it take to drive your laser, and how small could it be made? Olivier:

Our laser is about $0.5 \%$ efficient, so to get $1.5 \mathrm{kw}$ of $589 \mathrm{~nm}$ light takes a fairly large amount of power. We eventually expect to field $\mathrm{a} \sim 100 \mathrm{~W}$ laser at an observatory which would fit in a small room or on the back of a truck.

Elias:

Have you shown how laser guide stars will be applied to astronomical imaging? Are there plans to use laser guide stars in atmospheric studies and if so, what will these studies be?

Olivier:

It is usually better to use natural stars to study atmospheric turbulence; however, our laser is being used as a powerful lidar source to study atmospheric chemistry.

Napier:

Would it be possible to extend this technique to provide phase calibration sources for millimeter or submillimeter wavelength arrays? This would involve exciting atmospheric molecules with a laser and having them re-emit at $\mathrm{mm}$ or sub-mm wavelengths rather than, or as well as, optical wavelengths.

Olivier:

In principle, this should be possible. I don't know whether it is technically feasible. 